\title{
PRESENCIA DE MACRORRESTOS PARAUTÓCTONOS DE Sabalites cf. longirhachis (UNGER, 1850) KVAČEK \& HERMAN, 2004 EN FACIES PARÁLICAS DEL CRETÁCICO SUPERIOR DEL PIRINEO ORIENTAL
}

\author{
Josep MARMI ${ }^{1,2}$, Bernard GOMEZ ${ }^{3}$ y Carles \\ MARTÍN-CLOSAS \\ ${ }^{1}$ Institut Català de Paleontologia, C/ Escola Industrial 23, E-08201 Sabadell \\ (Barcelona).josep.marmi@icp.cat \\ ${ }^{2}$ Consorci Ruta Minera, Carretera de Ribes 20, E-08698 Cercs (Barcelona) \\ ${ }^{3}$ CNRS-UMR 5125, Paléoenvironnements et Paléosphère, Université Lyon 1, \\ 43 Bd du 11 Novembre 1918, F-69622 Villeurbanne cedex, France. \\ bernard.gomez@univ-lyon1.fr \\ ${ }^{4}$ Departament d'Estratigrafia, Paleontologia i Geociències Marines, Universitat \\ de Barcelona, c/ Martí i Franquès s/n, E-08028 Barcelona. \\ cmartinclosas@ub.edu.
}

\begin{abstract}
Marmi, J., Gomez, B. \& Martín-Closas, C. 2008. Presencia de macrorrestos parautóctonos de Sabalites cf. longirhachis (Unger, 1850) Kvaček \& Herman, 2004 en facies parálicas del Cretácico Superior del Pirineo oriental [Occurrence of parauthocthonous megaremains of Sabalites cf. longirhachis (Unger, 1850) Kvaček \& Herman, 2004 in paralic facies from the Upper Cretaceous of the Eastern Pyrenees.] Revista Española de Paleontología, 23 (1), 7-14. ISSN 0213-6937.
\end{abstract}

\begin{abstract}
Costapalmate leaf adpressions of Sabalites cf. longirhachis have been recently discovered from the Upper Cretaceous sites of Fumanya (Berguedà) and Pinyes (Alt Urgell) in the Eastern Pyrenees. Moreover, the Fumanya outcrops supply significant data for the habitat of this plant in brackish coastal swamps.
\end{abstract}

Keywords: Arecaceae, Sabalites, Fumanya, Eastern Pyrenees, Maastrichtian.

RESUMEN

En los yacimientos del Cretácico Superior de Fumanya (Berguedà) y Pinyes (Alt Urgell), en el Pirineo Oriental, se han descubierto recientemente adpresiones de hojas costapalmadas atribuidas a Sabalites cf. longirhachis. El yacimiento de Fumanya proporciona datos relevantes sobre el hábitat de esta planta en marismas costeras salobres.

Palabras clave: Arecaceae, Sabalites, Fumanya, Pirineo Oriental, Maastrichtiense.

\section{INTRODUCCIÓN}

Los restos más antiguos de palmeras fósiles consisten en hojas costapalmadas del Coniaciense-Campaniense inferior de América del Norte y Europa (Berry, 1905, 1911, 1914; Kvaček \& Herman, 2004). Desde finales del Cretácico superior la mayoría de taxones dentro de Arecaceae, familia de monocotiledóneas que incluye las palmeras, ya están presentes en el registro fósil (Harley, 2006). En general, las monocotiledóneas son relativamente escasas en el registro de macroflora, siendo más abundantes en las pali- nofloras. Sin embargo, los restos fósiles de hojas, troncos, raíces, frutos y semillas atribuidos a la familia Arecaceae están bien documentados en la literatura (ver revisión en Harley, 2006). En un artículo reciente sobre la flora de la Formación Grünbach (Alpes orientales, Austria) se documentan unos de los restos más antiguos de palmeras en Europa (Kvaček \& Herman, 2004). Se trata de restos de hojas costapalmadas del Campaniense inferior, clasificados como Sabalites longirhachis (Unger, 1850) Kvaček \& Herman, 2004. Los mismos autores sugieren incluir dentro de este parataxon a Juranyia hemiflabellata Tuzson, 1908, 
del Maastrichtiense de Rumania, ya que presenta también morfología costapalmada. En el sur de Francia Sabalites longirhachis (Unger, 1850) Kvaček \& Herman, 2004 fue citado previamente en el Campaniense-Maastrichtiense de la localidad de Fuveau (Saporta, 1890) y, en el Pirineo Oriental, ha sido citado en las cercanías del pueblo de Isona (cuenca de Tremp) a partir de material fragmentado (Vicente, 2002).

Sabalites longirhachis (Unger, 1850) Kvaček \& Herman, 2004 representaría la forma primitiva a partir de la cuál pudieron evolucionar las hojas pinnadas y palmadas (Kvaček \& Herman, 2004). Esta forma estaría caracterizada por presentar una morfología costapalmada, induplicada, simple y con segmentos foliares totalmente fusionados o, a veces, mínimamente diseccionados en su parte apical (Kvaček \& Herman, 2004). Aunque no se conoce con certitud el pariente vivo más cercano de Sabalites, se ha sugerido, en base a datos moleculares, que la subfamilia Coryphoideae, que contiene palmeras con hojas costapalmadas, seria uno de los grupos basales dentro de Arecaceae (Lewis \& Doyle, 2001). Sin embargo, otros estudios moleculares indican que Coryphoideae sería el grupo hermano de las subfamilias Arecoideae y Ceroxyloideae, quedando sólo Nypoideae y Calamoideae como grupos basales (Hann, 2002; Asmussen et al., 2006).

Recientemente, se han descubierto impresiones de hojas de palmeras costapalmadas en los yacimientos del área de Fumanya, en los municipios de Fígols y Vallcebre (Berguedà, Barcelona) y de Pinyes, en el municipio de Coll de Nargó (Alt Urgell, Lleida) (Fig. 1). En el presente trabajo describimos la morfología de estos especimenes y presentamos por primera vez una hipótesis tafonómica y autecológica sobre el hábitat de Sabalites longirhachis (Unger, 1850) Kvaček \& Herman, 2004.

\section{CONTEXTO GEOLÓGICO}

Los yacimientos de Fumanya (sinclinal de Vallcebre) fueron descubiertos en 1985 (Viladrich, 1986) y consisten en cuatro afloramientos de capas subverticales donde se pueden observar millares de icnitas y varias decenas de rastros asignados a saurópodos titanosaurios (véase referencias en Vila et al., 2005). Los afloramientos donde se han localizado las impresiones de hojas son Fumanya Sur y Mina Esquirol. Ambas localidades se localizan en los materiales basales de la Formación Tremp (Mey et al., 1968), dentro del clásicamente llamado "Garumniense" gris (Rosell et al., 2001). En el sinclinal de Vallcebre, los restos vegetales estudiados se incluyen en la parte superior de la unidad "Calizas margosas" conocida como "cementos", de acuerdo con Aepler (1967) y Rosell et al. (2001), y que presenta una edad Maastrichtiense inferior (Oms et al., 2007) (Fig. 2). Además de las calizas margosas, la unidad regional del Garumniense gris presenta una alternancia de calizas lacustres con margas orgánicas y carbones (lignitos) que se depositó bajo condiciones reductoras, en un ambiente de marisma supra-mareal (Rosell et al., 2001). La presencia de carofitas de aguas salobres (poblaciones homogéneas de Feistiella sp.) en niveles inmediatamente

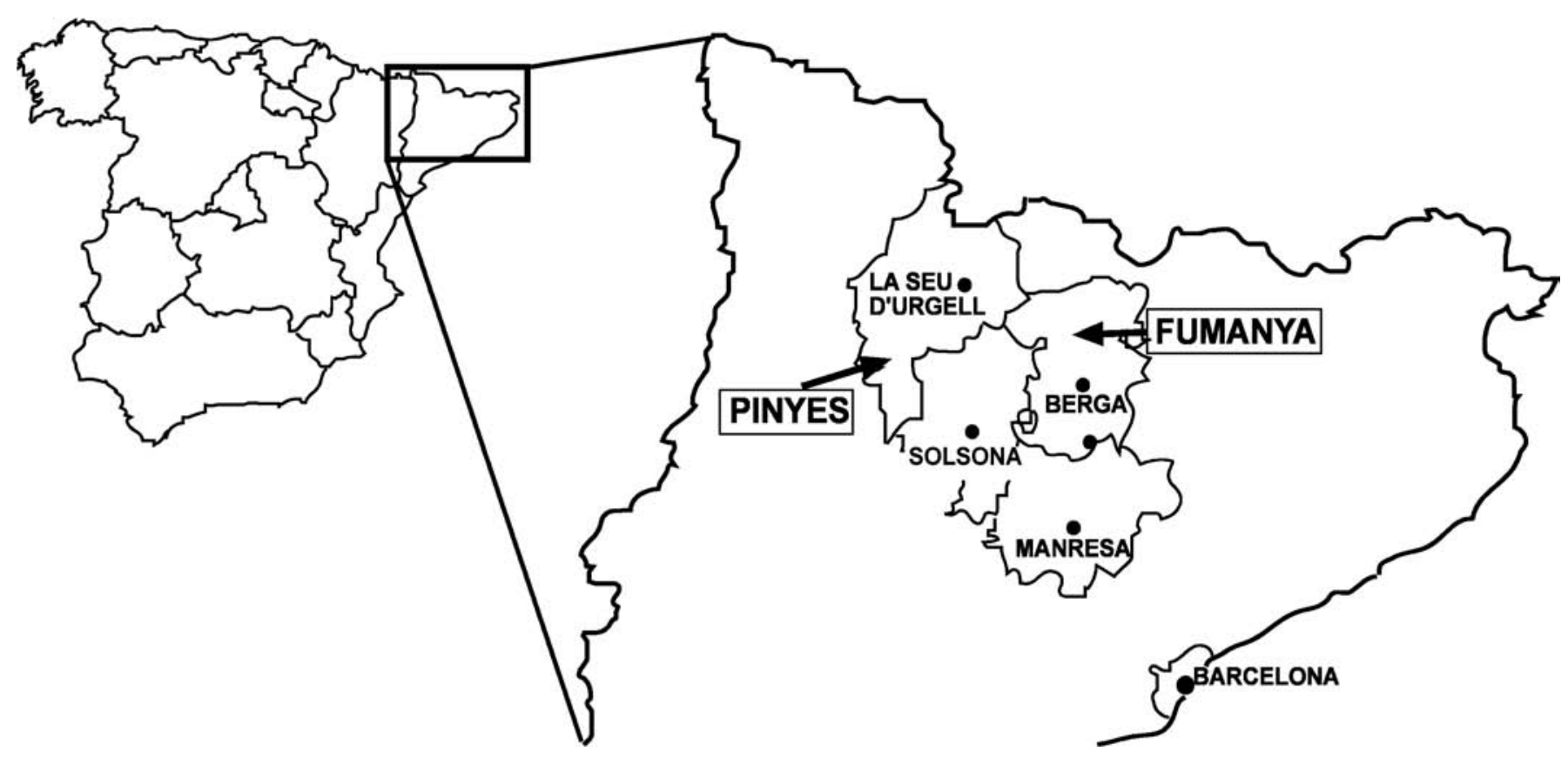

Figura 1. Localización geográfica de los yacimientos de Fumanya y Pinyes.

Geographic location of Fumanya and Pinyes sites. 


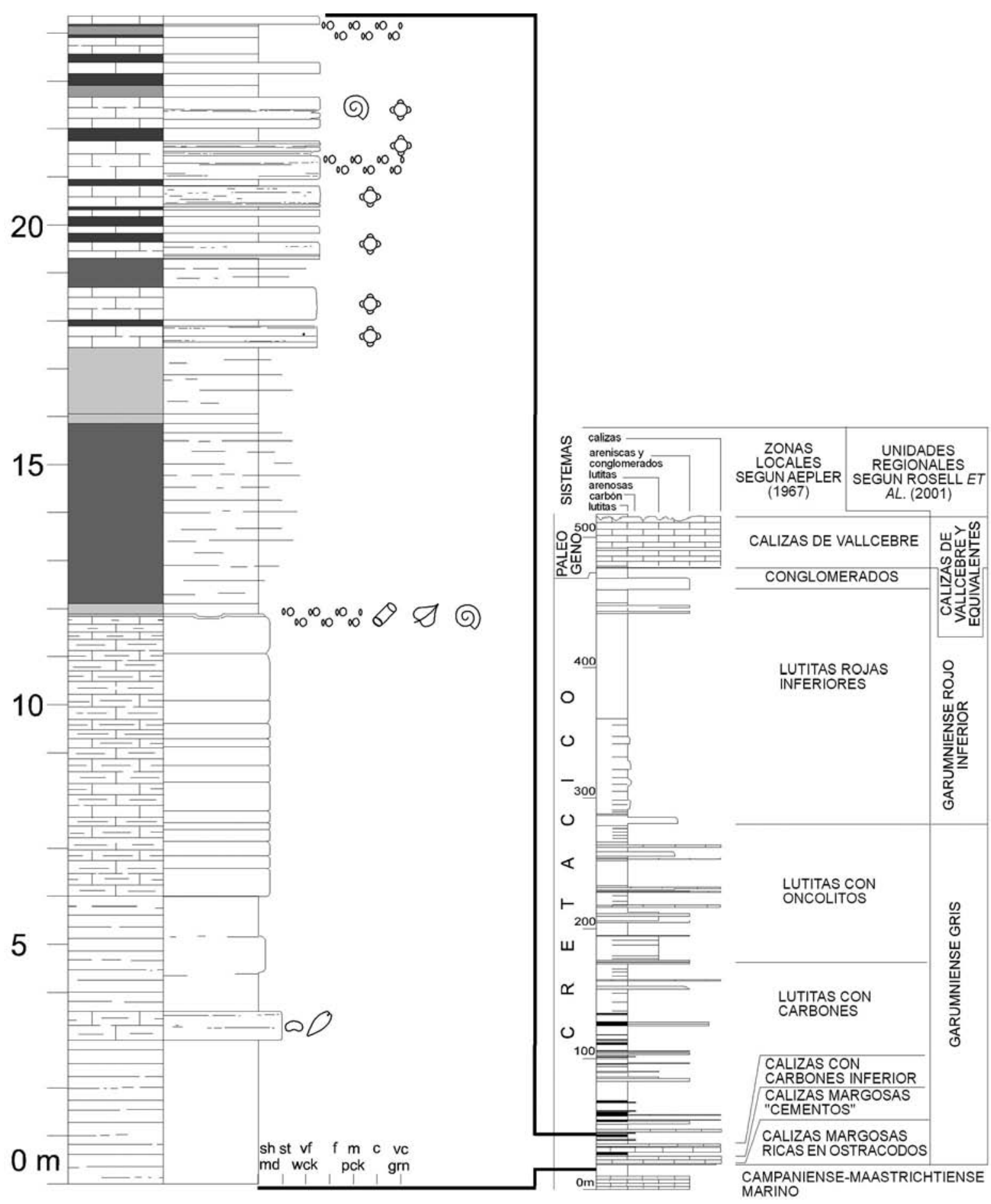

\section{LEYENDA}

lutitas oscuras

y lignitos

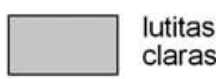

claras

areniscas

claras
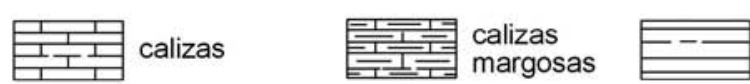

margas

carófitas $\oslash$ impresiones de hojas de Sabalites

$\&$ burrowing $\bigcirc$ bivalvos gasterópodos

ostrácodos $\quad{ }^{10} 10^{10} .0^{\circ} .0^{\circ}$ icnitas de dinosaurio

Figura 2. Serie estratigráfica sintética de Fumanya (modificada de Vila et al., 2005) con la localización del nivel rico en hojas de Sabalites cf. longirhachis (Unger, 1850) Kvaček \& Herman, 2004.

Synthetic stratigraphy of Fumanya (modified from Vila et al., 2005) with the location of the level rich in leaves of Sabalites cf. longirhachis (Unger, 1850) Kvaček \& Herman, 2004. 
suprayacentes a los que han proporcionado las hojas de palmeras sugieren que en la marisma predominaban las condiciones salobres. Feist \& Colombo (1983) también señalan la presencia de una fauna de ostrácodos salobres en estos niveles (Cyprideis sp., Neocyprideis durocortoriensis Apostolescu, 1956). La presencia de niveles monopespecíficos de Corbicula laletana Vidal, 1874 viene a apoyar igualmente el carácter salobre de la sucesión.

El yacimiento de Pinyes es conocido por su abundante registro de huevos y puestas de dinosaurios (Escuer et al., 2003). En el área de Coll de Nargó los restos vegetales se incluyen en el tramo de calizas basales y margas grises, con niveles de carbones. Estratigráficamente se encuentran por encima de los materiales costeros de la Formación Areniscas de Arén. Su edad podría variar entre Campaniense superior y Maastrichtiense inferior (Feist \& Colombo, 1983).

\section{PALEONTOLOGÍA SISTEMÁTICA}

\author{
Clase LILIOPSIDA Scopoli, 1760 \\ Orden ARECALES Bromhead, 1840 \\ Familia Arecaceae Schultz-Schultzenstein, 1832 \\ Género Sabalites Saporta, 1865

\section{Sabalites cf. longirhachis (Unger, 1850) Kvaček \& Herman, 2004} \\ Fig. 3, Tab. 1
}

Material: Los ejemplares estudiados son los siguientes: BFUM15 (IPS-41681), impresión de lámina conservada casi totalmente (Fig. 3a); B-FUM16 (IPS-41682), impresión de hoja completa, incluyendo el pecíolo (Fig. 3b); V-FUM86, impresión parcial de lámina y de un fragmento de pecíolo (Fig. 3c); V-FUM137, impresión de unas 3/4 partes de la lámina y pecíolo (Fig. 3d); V-FUM185, impresión bastante erosionada de la mitad basal de la lámina y una parte del pecíolo (Fig. 3e); V-FUM223, impresión parcial de lámina donde la parte más completa corresponde a la mitad de la base (Fig. 3f); IPS-41683, impresión de la parte basal de la lámina y parte del pecíolo (Fig. 3g). Las impresiones originales se encuentran en los yacimientos de Mina Esquirol (B-FUM15, B-FUM16, V-FUM223), Fumanya Sur (V-FUM86, V-FUM137, V-FUM185) y Pinyes (sin catalogar). Algunos de los ejemplares, especialmente en los afloramientos de Fumanya, no se han excavado completamente debido a su fragilidad, pues su extracción supondría su destrucción y la exposición total a la intemperie puede suponer su pérdida irreversible por erosión a corto-medio plazo. Se han realizado réplicas de los especimenes de Fumanya (B-FUM15 y B-FUM16) y de Pinyes, que se encuentran depositadas en el Institut Català de Paleontologia bajo las siglas (IPS-41681, IPS-41682 y IPS41683 respectivamente). El molde se realizó con silicona y fibra de vidrio y la réplica con resina soluble en agua. Las medidas se han realizado directamente en las impresiones excepto en VFUM185 y V-FUM223, recurriendo en estos casos a la ayuda de fotografías debido a las dificultades de acceso. Los ángulos de inserción de los segmentos se han medido a partir de fotografías en todos los casos.

Descripción: Las hojas son costapalmadas y ovalo-lanceoladas. Las láminas más completas (Fig. 3a y b) miden entre 43 y $75 \mathrm{~cm}$ de longitud y entre 10 y $41 \mathrm{~cm}$ de ancho. Están formadas por entre unos 25 (B-FUM16) y 30 (B-FUM15) pares de segmentos foliares opuestos, induplicados (su sección transversal tiene forma de V), rectilíneos (o ligeramente curvados en la base) y totalmente fusionados o, a veces, ligeramente diseccionados en los márgenes (Fig. 3a y b). Los segmentos que nacen en la parte basal de la lámina son más delgados que los de las partes medial y apical. En B-FUM15 miden un promedio de $44 \mathrm{~cm}$ y en B-FUM16 unos $20 \mathrm{~cm}$ de longitud mientras que la anchura de los segmentos varía entre 0,35 y $1,4 \mathrm{~cm}$. Los segmentos pueden ser más estrechos en su base que en los márgenes de la lámina (Fig. 3a), se unen en la costa formando un ángulo agudo (entre $10-30^{\circ}$ en hojas pequeñas y entre 30 y $50^{\circ}$ en las hojas de mayor tamaño) y son ligeramente decurrentes en la parte apical de esta estructura (Fig. 3a). La costa es larga (hasta 35 $\mathrm{cm}$ de longitud), delgada y se introduce hasta aproximadamente la mitad de la lámina (Fig 3a y b). En los segmentos se puede distinguir un nervio central y otros de órdenes inferiores paralelos, a cada lado del principal. El pecíolo tiene más de medio metro de longitud y puede superar la longitud de la lámina (Fig. 3b). Puede presentar finas estriaciones longitudinales (Fig. 3d). Las medidas efectuadas en los diferentes especímenes se resumen en la Tabla 1.

Discusión: De acuerdo con Read \& Hickey (1972), el género Sabalites incluye hojas de arecáceas de morfología costapalmada. Sin embargo este tipo de morfología también aparece en otros géneros como el extinto Geonomites Lesquereux, 1878 (Campaniense) y el actual Sabal Adanson, 1763. A falta de una revisión detallada, el género Geonomites se diferencia de Sabalites en caracteres sutiles como la mayor longitud de las bases decurrentes de los segmentos foliares al unirse a la costa. Las diferencias

Figura 3. Sabalites cf. longirhachis (Unger, 1850) Kvaček \& Herman, 2004 del Cretácico Superior del Pirineo Oriental. Impresiones de las hojas estudiadas en este trabajo. a, B-FUM15 (IPS-41681); b, B-FUM16 (IPS-41682); c, V-FUM86; d, V-FUM137; e, V-FUM185; f, V-FUM223; g, IPS-41683. Las escalas son las siguientes: $5 \mathrm{~cm}$ en a y c; $15 \mathrm{~cm}$ en b y f; $6 \mathrm{~cm}$ en d; 25 $\mathrm{cm}$ en e. La brocha de $\mathbf{g}$ mide $20 \mathrm{~cm}$.

Sabalites $c f$. longirhachis (Unger, 1850) Kvaček \& Herman, 2004 from the Upper Cretaceous of Eastern Pyrenees. Impressions of leaves studied. a, B-FUM15 (IPS-41681); b, B-FUM16 (IPS-41682); c, V-FUM86; d, V-FUM137; e, V-FUM185; $\boldsymbol{f}$, V-FUM223; $\boldsymbol{g}$, IPS-41683. The scale bars correspond to: $5 \mathrm{~cm}$ in $\boldsymbol{a}$ and $\boldsymbol{c} ; 15 \mathrm{~cm}$ in $\boldsymbol{b}$ and $\boldsymbol{f} ; 6 \mathrm{~cm}$ in $\boldsymbol{d} ; 25 \mathrm{~cm}$ in $\boldsymbol{e}$. The paintbrush from $\mathrm{g}$ is $20 \mathrm{~cm}$ long. 


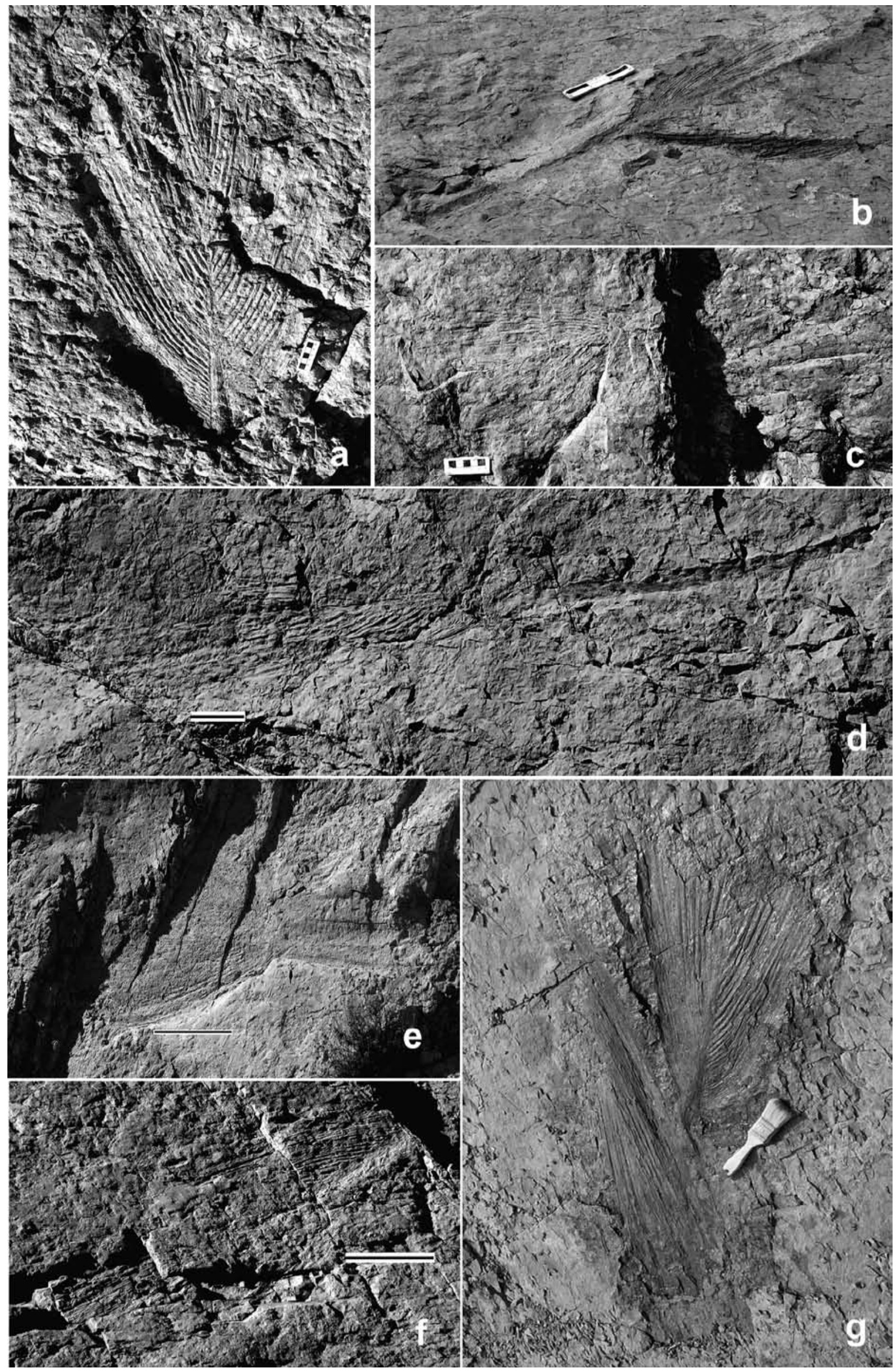




\begin{tabular}{|c|c|c|c|c|c|c|c|}
\hline & $\begin{array}{c}\text { B-FUM15 } \\
\text { (IPS-41681) }\end{array}$ & $\begin{array}{c}\text { B-FUM16 } \\
\text { (IPS-41682) }\end{array}$ & V-FUM86 & V-FUM137 & V-FUM185 & V-FUM223 & IPS-41683 \\
\hline Longitud total $(\mathrm{cm})$ & - & 96 & - & - & - & - & - \\
\hline Longitud lámina $(\mathrm{cm})$ & $75^{*}$ & 43 & - & $50-60$ & - & - & - \\
\hline Anchura lámina $(\mathrm{cm})$ & 41 & 10 & - & - & 60 & - & - \\
\hline Longitud pecíolo $(\mathrm{cm})$ & - & 53 & - & - & - & - & - \\
\hline Anchura pecíolo (cm) & - & $2,15-4,7$ & 5,6 & 2,5 & - & - & - \\
\hline Longitud costa $(\mathrm{cm})$ & 30 & 15 & 10,3 & 20 & - & - & 35 \\
\hline Anchura costa en la base $(\mathrm{cm})$ & 3,6 & 2,0 & - & 1,85 & - & - & 3,2 \\
\hline Longitud segmentos $(\mathrm{cm})$ & $42-47$ & $18-22$ & - & - & - & - & - \\
\hline Anchura segmentos $(\mathrm{cm})$ & $1,0-1,4$ & $0,35-0,6$ & $0,4-0,8$ & 0,6 & - & - & $0,4-1,0$ \\
\hline Ángulo segmentos-costa & $30^{\circ}-50^{\circ}$ & $10^{\circ}-25^{\circ}$ & $25^{\circ}-30^{\circ * *}$ & $10^{\circ}-30^{\circ}$ & - & $30^{\circ * * *}$ & $15^{\circ}-45^{\circ}$ \\
\hline
\end{tabular}

Tabla 1. Valores de las medidas efectuadas en las hojas costapalmadas de los yacimientos de Fumanya y Pinyes. Leyenda: * lámina casi completa; $* *$ en segmentos de la mitad apical y parte medial de la costa; $* * *$ en segmentos de la parte medial de la costa.

Size values of costapalmate leaves from Fumanya and Pinyes sites. Caption: *almost complete lamina, **in segments from half apical and medial part of costa, *** in segments from the medial part of costa.

respecto a Sabal son más evidentes ya que en este género los segmentos están profundamente diseccionados mientras que en Sabalites están fusionados prácticamente hasta los márgenes de la lámina. Ninguno de los caracteres que diferencian Geonomites y Sabal está presente en los ejemplares de Fumanya y Pinyes. En Kvaček \& Herman (2004) se indican los principales caracteres morfológicos que pueden ser diagnósticos para distinguir el parataxón estudiado en este trabajo de las paraespecies descritas en el Cretácico Superior y Paleógeno de América del Norte. Sabalites carolinensis Berry, 1914, del Santoniense de Carolina del Sur (Estados Unidos) presenta una costa corta y segmentos estrechos en forma de cuña y divididos en los extremos. Sabalites ungeri (Lesquereux, 1878) Dorf, 1939, del Paleoceno de Nuevo México (Estados Unidos) presenta una costa robusta y estriada. Las otras especies paleógenas aceptadas por Read \& Hickey (1972) se caracterizan por tener una costa muy corta en comparación con Sabalites longirhachis (Unger, 1850) Kvaček \& Herman, 2004, según (Kvaček \& Herman, 2004). Sabal dortchii Daghlian, 1978, del Eoceno de Tennessee (Estados Unidos) presenta una costa alargada pero puede diferenciarse de Sabalites a partir de características de la cutícula.

Las características morfológicas de los especimenes descritos en este trabajo coinciden en su totalidad con la mayoría de caracteres diagnósticos de Sabalites longirhachis (Unger, 1850) Kvaček \& Herman, 2004. La diagnosis de esta especie se basa en: (1) hojas costapalmadas, induplicadas y lanceoladas; (2) hojas con costa larga y estrecha; (3) segmentos fusionados, algunas veces ligeramente diseccionados en los márgenes de la hoja, rectilíneos, ligeramente más anchos hacia el ápice, uniéndose a la costa en un ángulo agudo y unidos decurrentemente a la parte apical de esta estructura (Kvaček \& Herman, 2004). Según estos autores, la diagnosis de Sabalites longirhachis también se basa en caracteres histológicos y de morfología celular: cada segmento tiene un nervio principal central y otros paralelos a este organizados en cuatro órdenes diferentes, las cutículas abaxial y adaxial tienen células alongadas y la cutícula abaxial estomas tetracíticos. Las convergencias morfológicas son menos probables en estos últimos caracteres pero hasta el momento no se dispone de detalles cuticulares en los ejemplares del Pirineo. En este sentido, de acuerdo con los caracteres morfológicos disponibles, asignamos los ejemplares descritos en este trabajo a Sabalites cf. longirhachis.

\section{TAFONOMÍA Y PALEOAUTECOLOGÍA}

Los especimenes de Fumanya representan el material más completo a nivel macroscópico de este parataxón descrito hasta el momento. Los ejemplares descritos por Vicente (2002) en Isona y por Kvaček \& Herman (2004) en Austria son fragmentados y en ningún caso conservan el pecíolo en conexión anatómica con el limbo. Es muy probable que las partes que faltan en los ejemplares estudiados [excepto B-FUM16 (IPS-41682), que se ha conservado en su totalidad] se hayan perdido por erosión y que originalmente las hojas fosilizaran enteras. Desde el punto de vista tafonómico la presencia de la hoja entera, incluyendo lámina y pecíolo en conexión anatómica, son muy significativos ya que la mayoría de palmeras presentan hojas marcescentes, que se degradan sobre la 
planta una vez muertas, incluso recubriendo el tronco en su totalidad, lo que dificulta su entrada en el registro fósil en un buen estado de conservación (Martín-Closas \& Gomez, 2004). La conservación de la hoja entera indicaría un tipo de producción tafonómica traumática y un hábitat cercano al ambiente deposicional (marisma litoral salobre).

Otro dato interesante de las impresiones de hojas de Fumanya es la posible conservación de diversos estadios de crecimiento. A partir de las dimensiones de la lámina, de la costa y de los ángulos de inserción de los segmentos a la costa (Tabla 1), se sugiere que las impresiones B-FUM16 (IPS-41682), V-FUM86 y V-FUM137 corresponderían a hojas jóvenes y el resto a hojas adultas. La conservación de diferentes estadios de crecimiento en el mismo nivel viene a apoyar la hipótesis de un hábitat cercano al medio deposicional para los restos estudiados.

\section{CONCLUSIONES}

Se describe la presencia de hojas costapalmadas muy bien conservadas de Sabalites cf. longirhachis en el Campaniense y Maastricthiense del Pirineo oriental. Estas hojas representan el macrorresto más completo hallado hasta el momento de este parataxón. Los fósiles son parautóctonos en los niveles de margas intercaladas con lignitos de la Formación Tremp en los afloramientos de Fumanya, Mina Esquirol y Pinyes. Estos niveles se han atribuido a facies de marisma parálica salobre, en las que se sugiere que viviría la planta.

\section{AGRADECIMIENTOS}

El trabajo de campo fue posible gracias al permiso de prospección concedido por la Direcció General de Patrimoni Cultural (Departament de Cultura i Mitjans de Comunicació, Generalitat de Catalunya). Este estudio ha sido financiado por los proyectos 2006EXCAVA0001 (Generalitat de Catalunya), CGL2005-00046, CGL2005-01121 y CGL2005-00404 (Ministerio de Educación y Ciencia). Agradecemos al Dr Ángel Galobart (Institut Català de Paleontologia), a Josep Peralba (Coll de Nargó), al Dr Oriol Oms (Universitat Autónoma de Barcelona) y a Bernat Vila (ICP) su ayuda en diversos aspectos de la realización del trabajo.

Josep Marmi es investigador postdoctoral (Programa Juan de la Cierva) del ICP.

\section{REFERENCIAS BIBLIOGRÁFICAS}

Adanson, M. 1763. Familles des Plantes, 2 vol., Paris, Vincent. Aepler, R. 1967. Das Garumnian der Mulde von Vallcebre und ihre Tektonik (Spanien, Provinz Barcelona). Master thesis der Freien Universität Berlin (Naturwissenschftlichen Fakultät), 101 pp. (inédito).
Asmussen, C.B., Dransfield, J., Deickmann, V., Barfod, A.S., Pintaud, J.C. \& Baker, W.J. 2006. A new subfamily classification of the palm family (Arecaceae): evidence from plastid DNA phylogeny. Botanical Journal of the Linnean Society, 151, 15-38.

Berry, E.W. 1905. A palm from the mid-Cretaceous. Torreya, 5, 30-33.

Berry, E.W. 1911. Contributions to the Mesozoic flora of the Atlantic coastal plain-VII. Butlletin of the Torrey Botanical Club, 38, 399-424.

Berry, E.W. 1914. The Upper Cretaceous and Eocene floras of South Carolina and Georgia. US Geological Survey Professional Paper, 84, 5-200.

Daghlian, C.P. 1978. Coryphoid palms from the lower and middle Eocene of southeastern North America. Palaeontographica $B, \mathbf{1 6 6}, 44-82$.

Dorf, E. 1939. Fossil plants from the Upper Cretaceous Aguja Formation of Texas. American Museum Novitates, 1015, 1-9.

Escuer, J., Peralba, J., Cusso, R., Jimenez, A., Borrull, J., Perez, P., Umbert, M., Garrabou, E., Bons, N., Garrabou, M. \& Regal, C. 2003. Sallent Valley, recurrent dinosaur nesting site from the Late Cretaceous of southern Pyrenees (Coll de Nargó, Spain): a GIS approach. Abstract 2nd Int. Symposium on Dinosaur Eggs and Babies, Montpellier, 11.

Feist, M. \& Colombo, F. 1983. La limite Crétacé-Tertiaire dans le nord-est de l'Espagne, du point de vue des charophytes. Géologie Méditerranéenne, 10, 303-326.

Hahn, W.J. 2002. A molecular phylogenetic study of the Palmae (Arecaceae) based on $a t p \mathrm{~B}, r b c \mathrm{~L}$, and $18 \mathrm{~S}$ nrDNA sequences. Systematic Biology, 51, 92-112.

Harley, M.M. 2006. A summary of fossil records for Arecaceae. Botanical Journal of the Linnean Society, 151, 39-67.

Kvaček, J. \& Herman, A.B. 2004. Monocotyledons from the Early Campanian (Cretaceous) of Grünbach, Lower Austria. Review of Palaeobotany and Palynology, 128 323-353.

Lesquereux, L. 1878. Contribution to the fossil flora of the Western Territories, Part II. The Tertiary flora. US Geological Survey Reports, VII, 1-366.

Lewis, C.E. \& Doyle, J.J. 2001. Phylogenetic utility of the nuclear gene Malate Synthase in the palm family (Arecaceae). Molecular Phylogenetics and Evolution, 19, 409-420.

Martín-Closas, C. \& Gomez, B. 2004. Taphonomie des plantes et interprétations paléoécologiques. Une synthèse. Geobios, 37, 65-888.

Mey, P.H., Nagtegaal, P.J.C., Roberti, K.J. \& Hartevelt, J.J.A. 1968. Lithostratigraphic subdivision of Post-Hercynian deposits in the South-Central Pyrenees, Spain. Leidse Geologische Mededelingen, 41, 221-228.

Oms, O., Dinarès-Turell, J., Vicens, E., Estrada, R., Vila, B., Galobart, À. \& Bravo, A.M. 2007. Integrated stratigraphy from the Vallcebre basin (southeastern Pyrenees, Spain): new insights on the continental Cretaceous-Tertiary transition in southwest Europe. Palaeogeography, Palaeoclimatology, Palaeoecology, 255, 35-47.

Read, R.W. \& Hickey, L.J. 1972. A revised classification of fossil palm and palm-like leaves. Taxon, 21, 129-137. 
Rosell, J., Linares, R. \& Llompart, C. 2001. El "Garumniense" Prepirenaico. Revista de la Sociedad Geológica de España, 14, 47-56.

Saporta, G. 1865. Études sur la vegetation du sud-est de la France a l'époque tertiaire. Annales des Sciences Naturelles, Botanique, 53, 1-152.

Saporta, G. 1890. Le Nelumbium provinciale des lignites crétacés de Fuveau en Provence. Mémoires de la Société Géologique de France, Paléontologie, 5, 1-10.

Tuzson, J. 1908. Adatok Magyarorszag fosszilis florajahoz (II). Additamenta ad floram fossilem Hungariae. Novenytani Kozlemenyek, 7, 1-4.

Unger, F. 1850. Genera et species plantarum fossilium.
Academia Caesareae Scientiarum, Wilhelm Braumueller, Vindobonae, $627 \mathrm{pp}$.

Vicente, J. 2002. Estudi morfológic de la flora cretácica d'Isona (Pallars Jussà). Institut d'Estudis de la Natura del Barcelonés Nord. Santa Coloma de Gramenet, 223 pp.

Vila, B., Oms, O. \& Galobart, A. 2005. Manus-only titanosaurid trackway from Fumanya (Maastrichtian, Pyrenees): further evidence for an underprint origin. Lethaia, 38, 211-218.

Viladrich, L. 1986. Les petjades fòssils del Coll de Fumanya. L'Erol, 18, 7-12.

Manuscrito recibido: 25 de Enero, 2007 Manuscrito aceptado: 12 de Diciembre, 2007 